\title{
Deep Learning for Sparse Scanning Electron Microscopy
}

Patrick Trampert ${ }^{1,2, *}$, Sabine Schlabach ${ }^{3}$, Tim Dahmen $^{1}$ and Philipp Slusallek ${ }^{1,2}$

1. German Research Center for Artificial Intelligence GmbH (DFKI), Saarbrücken, Germany.

2. Saarland University, 66123 Saarbrücken, Germany.

3. Karlsruhe Institute of Technology, 76131 Karlsruhe, Germany.

* Corresponding author: patrick.trampert@dfki.de

High-throughput scanning electron microscopy (SEM) has the goal to acquire large volumes at high resolution [1]. For samples of several millimeters a full scan with a traditional dense scanning pattern would take years. There are various ways to reduce the required time as has been evaluated in [2]. The authors showed that the best strategy for reducing overall acquisition times are sparse scanning methods that record only a small percentage of all possible pixels but with a reasonably high dwell time per pixel. The sparse data pattern must be reconstructed afterwards. As one solution missing data can be reconstructed using inpainting methods [2]. One promising approach is exemplar-based inpainting [3], which can deliver useful results without the introduction of artifacts that have not been observed in known data, as only prior knowledge of already fully scanned images is used for the reconstruction process.

Inspired by [4], we investigated into a new approach that uses deep learning for the reconstruction based on the sparse pattern. The discriminative feature in comparison to most known methods is that no prior knowledge is needed. Instead of starting from known image patches or dictionaries as used in sparse coding, the algorithm is initialized with a dense random pattern and the positions and pixel values of the scanned data. A convolutional neural network is trained based on this initialization. While training, the missing parts of the image are reconstructed. For the reconstruction of three-dimensional SEM acquisitions the procedure works iteratively slice by slice. The reconstruction of the first slice takes many iterations in the training process. However, after the first image has been reconstructed, the process is sped up. Due to the similarity of neighboring thin slices the networks parameters need not to be altered a lot, so that few iterations in general suffice for subsequent reconstructions.

As proof-of-concept we evaluated the algorithm based on SEM acquisitions of filled micro silica balls (Figure 1). Sparse scans were simulated with $1 \%, 5 \%$, and $20 \%$ of the original data. We reconstructed the sparse data using random data as initialization and computed peak-signal-to-noise-ratio (PSNR) and structural similarity index (SSIM) for each experiment, as depicted in Figure 1. The results of our experiments are very promising and further research into the capabilities of deep learning for the reconstruction of sparsely sampled data must be conducted.

Summarizing, deep learning offers a great potential for high-throughput SEM. Besides segmentation, which has been investigated a lot, even in electron microscopy use cases, our proposed procedure does not need any manually generated labels to be used in a supervised learning manner. Especially this independence of any prior knowledge may foster further applications [5]. 


\section{References:}

[1] F Bourghorbel et al., Microscopy and Microanalysis (23) (S1) (2017), p. 150.

[2] P Trampert et al., Ultramicroscopy 191 (2018) p. 11.

[3] P Trampert et al., Microscopy and Microanalysis 24 (S1) (2018) p.700.

[4] D Ulyanov et al., IEEE CVPR (2017).

[5] Research has been supported by Thermo Fisher Scientific. We thank the Repos project (BMBF) for provided images and U. Tallarek and his team at Uni Marburg for the samples. The authors thank the DFKI GmbH for additional funding and for providing the necessary infrastructure.
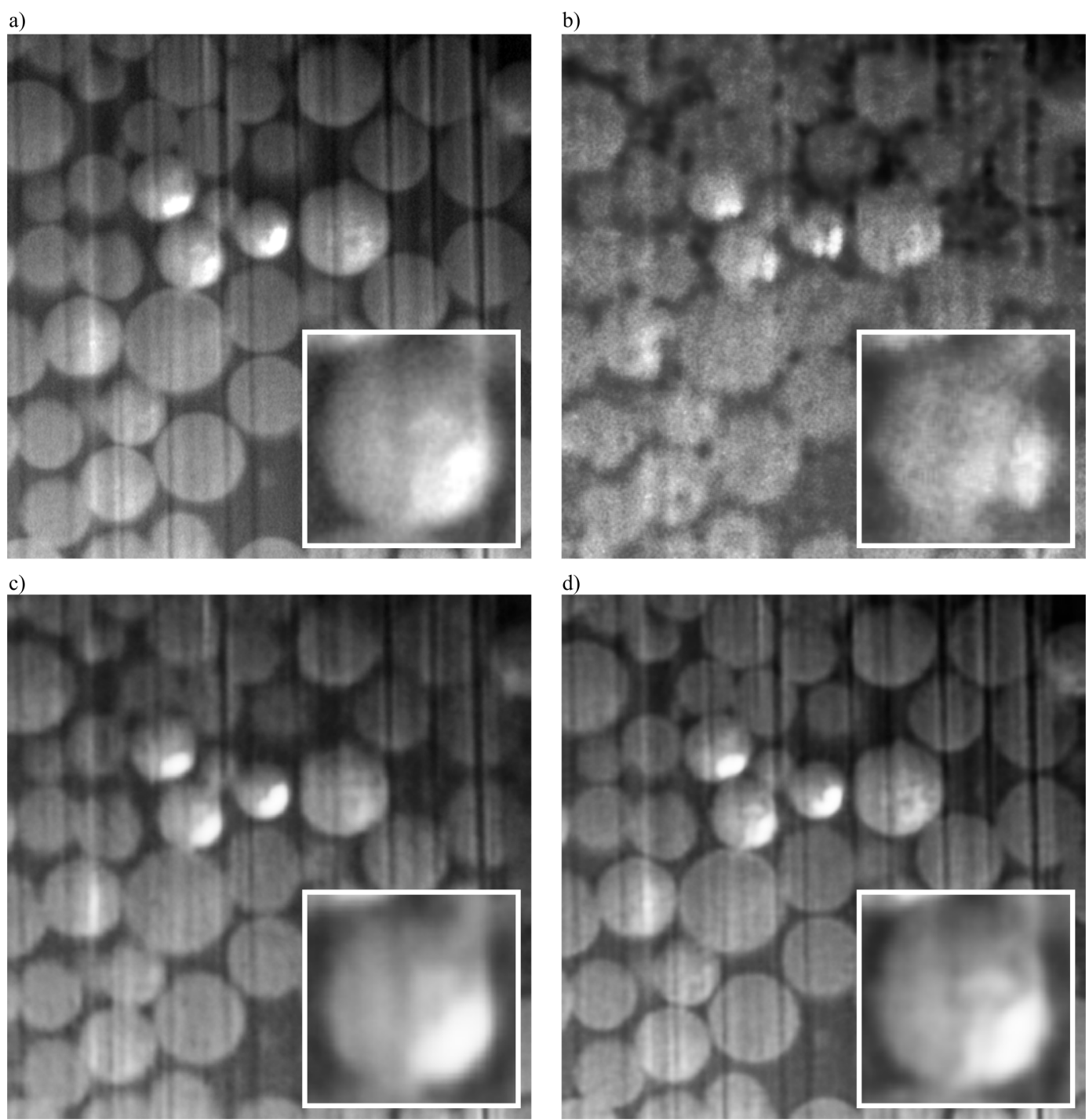

Figure 1. Silica micro balls excerpt and zoom in. a) Ground truth image. b) Reconstruction with $1 \%$ of original data. Sparse data acquisition was simulated with random sampling. PSNR=23.95, SSIM=0.60. c) Reconstruction with 5\%. PSNR=31.11, SSIM=0.82. d) Reconstruction with 20\%. PSNR=34.52, SSIM=0.87. 